\title{
Angina pectoris treated by ventricular plication
}

\author{
HUON H GRAY, MATHIAS PANETH, DEREK G GIBSON \\ From the Brompton Hospital, London
}

SUMMARY Objective analysis of the left ventricular angiogram in a patient with angina but normal coronary arteries showed an appreciable disturbance of regional wall movement. Because of persistent symptoms refractory to medical treatment left ventricular plication was undertaken. This resulted in a return to normal of a series of disturbances of left ventricular wall motion commonly found in patients with obstructive coronary disease, and a striking improvement in the patient's symptoms. The patient remains symptom free five years after operation.

It is well recognised that typical angina can occur when coronary arteriography is entirely normal, though in some patients abnormal left ventricular wall movement can be demonstrated by objective analysis of cineangiograms. We have previously argued that regional abnormalities of diastolic wall movement may interfere with coronary blood flow and so be the direct cause of anginal pain. ${ }^{1}$ Ventricular surgery, by modifying the pattern of wall movement, might thus be beneficial when intractable angina proves resistant to conventional medical treatment.

We report a patient with normal coronary arteries and abnormal left ventricular wall movement, in whom the chest pain resolved, exercise tolerance increased, and regional left ventricular wall motion was strikingly improved after left ventricular plication. He remains symptom free five years after operation.

\section{Case report}

A 59 year old civil servant was admitted to his local hospital in January 1978 with a three year history of exertional chest pain and dyspnoea, with a severe episode on the day of admission. Myocardial infarction was diagnosed on the basis of raised concentrations of cardiac enzymes, though the electrocardiogram, which showed sinus rhythm, a normal QRS axis, and right bundle branch block, did not change. He made an uneventful recovery and was discharged, but subsequently he was referred to this hospital because of continuing exertional chest pain and dyspnoea.

Requests for reprints to Dr Derek G Gibson, Cardiac Department, Brompton Hospital, Fulham Road, London SW3 6HP.
There was no relevant past or family history. He had stopped smoking $\mathbf{8 0}$ cigarettes per day four years before. Clinical examination was unremarkable apart from a corneal arcus and wide splitting of the second heart sound because of right bundle branch block. Chest $x$ ray and routine haematology and biochemistry were normal apart from a slight increase in the concentration of fasting serum cholesterol $(7.8$ $\mathrm{mmol} / \mathrm{l})$. Selective coronary arteriography was normal and left ventricular end diastolic pressure was 14 $\mathrm{mm} \mathrm{Hg}$. Objective angiographic analysis is discussed below.

The patient continued to have disabling chest pain on exertion and dyspnoea over the next few years despite numerous adjustments to his drug treatment. These episodes of pain resulted in several admissions to hospital, but there was never any change in the electrocardiogram or cardiac enzymes. Because of the strong clinical impression of angina the patient had two further coronary arteriograms over the next three years. Both of these were normal and left ventricular wall movement on cineangiography was the same as before. Formal exercise testing was performed on two occasions according to the standard Bruce protocol with the patient being limited by chest pain and dyspnoea. Interpretation of ST segment changes was complicated by the presence of right bundle branch block. The mean rate-pressure product at maximum exercise was $15950 \mathrm{~mm} \mathrm{Hg}$ / min. Echocardiography, 24 hour ambulatory electrocardiographic recording, and respiratory function tests were unremarkable.

For reasons to be discussed, left ventricular apical plication was advised. At operation (MP) in April 1982 the external appearances of the left ventricle were normal. On full cardiopulmonary bypass a left ventricular vent was inserted via the apex of the left ventricle, and the apex, including its posterior and 


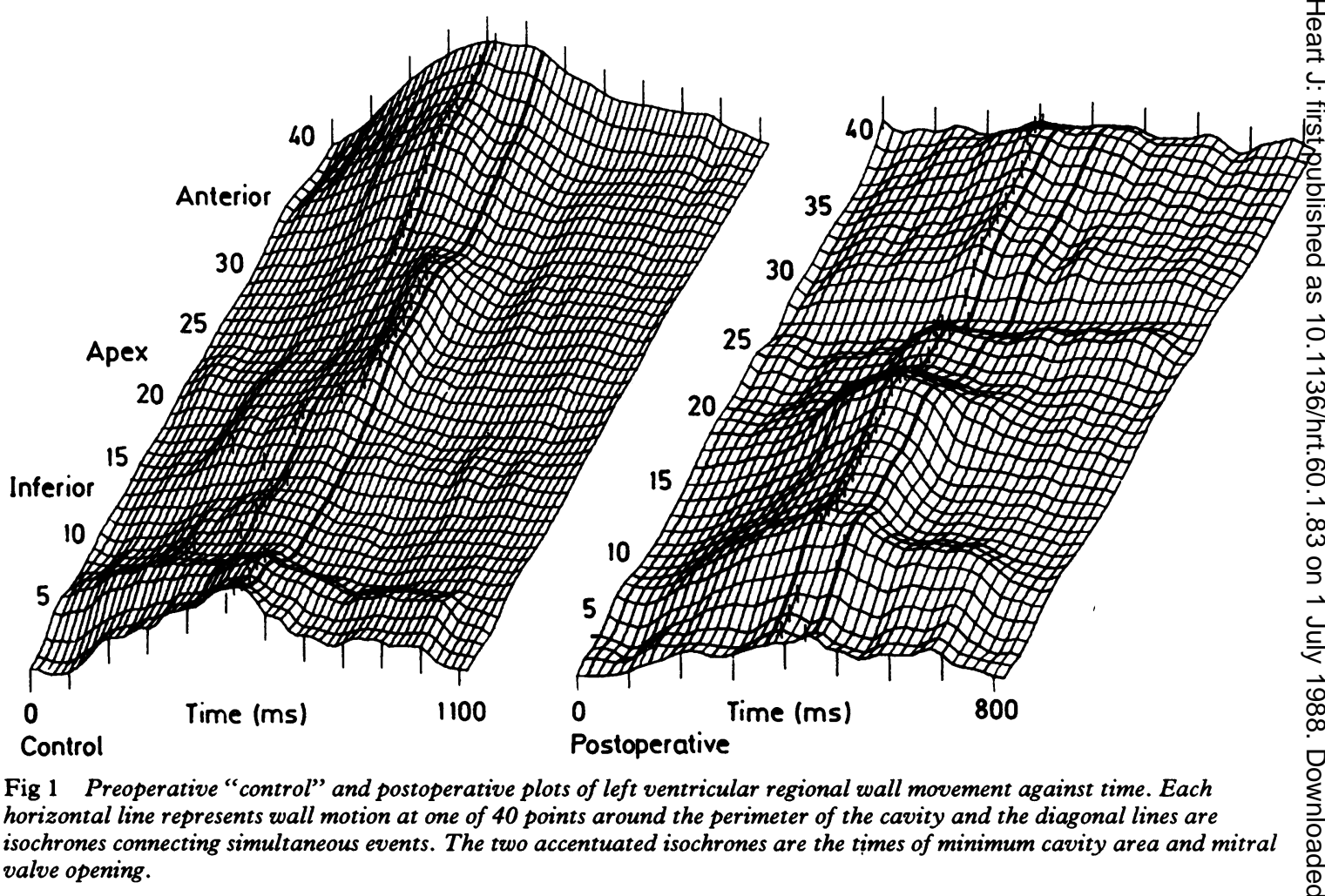

anterior walls, was plicated with mattress sutures of Ethibond tied over a U-shaped strip of Teflon felt. The heart was then defibrillated, the vent removed, and the site oversewn.

The patient's postoperative recovery was unremarkable and in five years of follow up he has been well with only very occasional episodes of left sided chest pain, considerable improvement in dyspnoea, and an increased exercise tolerance. Formal exercise testing has been performed twice since the operation and on both occasions this was limited by fatigue and the patient had no chest pain. The mean rate-pressure product at peak exercise was 28860 $\mathrm{mm} \mathrm{Hg} / \mathrm{min}$.

ANALYSIS OF LEFT VENTRICULAR ANGIOGRAMS The method underlying angiographic analysis ${ }^{2}$ is designed to display regional wall motion around the cavity outline throughout the cardiac cycle so that disturbances in the timing as well as the amplitude of regional wall motion can be studied and compared with normal results. ${ }^{3}$ The plots of regional wall motion (fig 1) continuously display the movement of each of forty points around the left ventricular cavity perimeter throughout the cardiac cycle. The same information can be presented as a contour display (fig 2). Each contour line represents $1 \mathrm{~mm}$ inward or outward motion of endocardium from its position in $\overrightarrow{\vec{B}}$ the end diastolic (maximum volume) frame an $\Phi$ shows the timing of such movement in relation to minimum cavity area and mitral valve opening.

The plots derived from the preoperative angiogram (November 1981) demonstrate an abnor mal reduction in the overall amplitude of inward motion along the inferior wall $\leqslant 4 \mathrm{~mm}$. On the anterior wall (segments 20-30) the onset of inward motion at the start of ejection was delayed by mores than $100 \mathrm{~ms}$, and this led to a characteristic pattern of "diagonal contours". In the same segments during the isovolumic relaxation period between minimumo cavity area and mitral opening there was abnorma inward motion of endocardium by $4 \mathrm{~mm}$, represent $f$ ing a significant change in left ventricular cavity shape. The preoperative ejection fraction was $0 \cdot 70$. N

The postoperative left ventricular angiogram $\left(\operatorname{Jan}_{\overline{0}} \mathrm{\omega}\right.$ uary 1983) showed major alterations from the preoperative pattern of regional wall motion. There was localised hypokinesis in segments $25-28$, corre sponding to the area plicated at operation, but the overall amplitude of left ventricular wall motion was normal along the entire inferior wall. In addition, the timing of wall motion was also normal. The onset of inward motion around the left ventricular cavity outline occurred within a period of $100 \mathrm{~ms}$ and the 

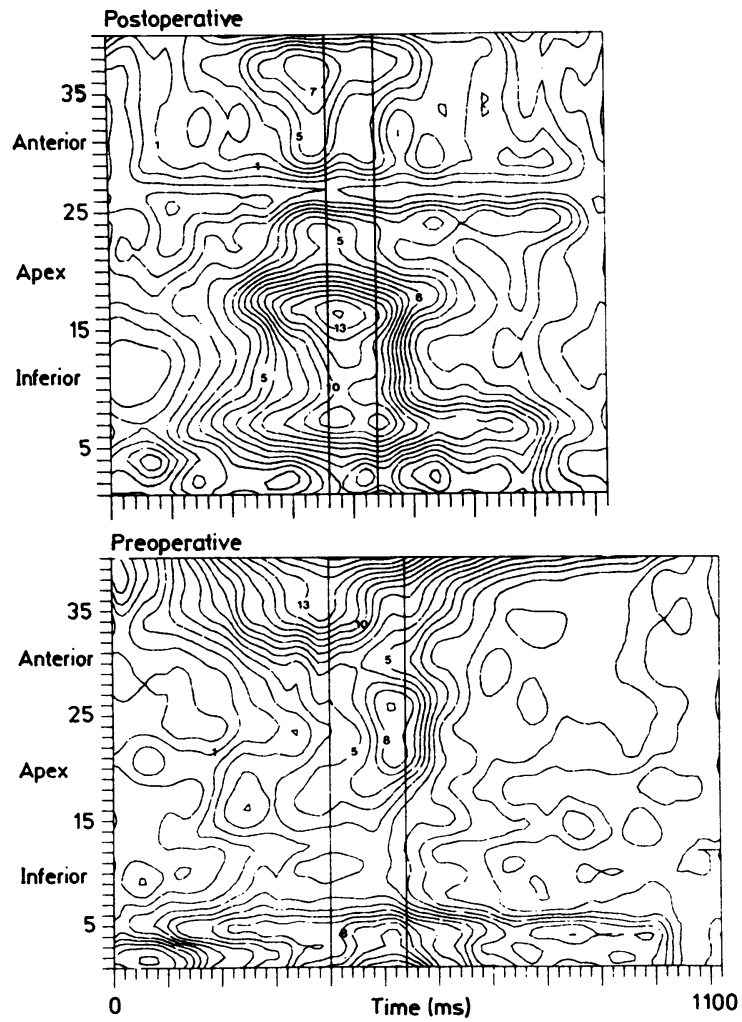

Fig 2 Preoperative and postoperative plots for the same data as fig 1 with left ventricular wall movement displayed as contours joining points moving in $1 \mathrm{~mm}$ increments from their position at maximum cavity area. The accentuated vertical lines are minimum cavity area and mitral valve opening.

disturbance of wall motion during isovolumic relaxation was no longer apparent. The ejection fraction $(0 \cdot 65)$ remained normal.

\section{Discussion}

The present case showed many of the familiar clinical features of the syndrome of chest pain with a normal coronary arteriogram. The patient required frequent admissions to hospital with episodes of chest pain that were believed to have been ischaemic in origin and there was objective limitation of exercise tolerance in terms of exercise load and rate-pressure product. Despite this, coronary arteriography was normal on three occasions. Arteriography was repeated because of the strong clinical impression that there must be an objective abnormality to account for the patient's severe and predictable limitation of exercise tolerance.

Although the large and medium sized coronary arteries were normal, the left ventriculogram showed several specific abnormalities, all of which may occur in patients with obstructive coronary artery disease. There was a major abnormality in the timing of wall motion during isovolumic relaxation that affected the free wall. ${ }^{2}$ In patients with single vessel disease this disturbance typically occurs in the territory supplied by the affected coronary artery and causes a characteristic change in ventricular cavity shape during the isovolumic period, with complementary outward motion elsewhere, usually along the upper part of the free wall as in the present case. ${ }^{2}$ Delay in the onset of inward wall motion at the start of ejection is also seen in patients with coronary artery disease under control conditions. Such regions are commonly the site of more pronounced disturbances of wall motion occurring during pacing induced angina, ${ }^{4}$ and in single vessel disease they again occur in the territory of the affected coronary artery.

Abnormal regional wall motion during isovolumic relaxation might of itself interfere with regional perfusion. Prolongation of local systole leads to a reduction in the time available for coronary flow, and in particular to loss of perfusion during the early diastolic period when coronary flow is usually rapid. ${ }^{5}$ Local ischaemia, once established, would be expected to cause further prolongation of systole and interference with relaxation, thus making the disturbance self-perpetuating and possibly explaining development of rest pain.

In view of these considerations, and of the manifest failure of medical treatment to control this patient's symptoms, we felt that operation was justified. After operation there was a striking improvement in symptoms with an increase in exercise tolerance and an $80 \%$ rise in rate-pressure product on exercise testing. It is now more than five years since operation and this improvement has been maintained.

The present case raises several questions. The patient had typical angina and detailed angiographic analysis showed at least three features commonly seen in patients with coronary artery disease, although coronary arteriography was normal. Coronary artery spasm is often invoked as an explanation, but it is difficult to see why any primary disturbance of coronary vasomotion should be so completely suppressed by localised plication of ventricular myocardium. A striking feature was the extent to which a widespread series of disturbances in regional left ventricular wall motion were reversed after local surgery. It would not have been unexpected for the abnormality of isovolumic relaxation to have been abolished, since the site of the plication itself was selected as that of the abnormal inward movement. More surprising is the reversion to normal of the preoperative inferior wall hypokinesia 
(an observation that excludes death of the myocardium as the basis for this abnormality) and the timing of onset of inward left ventricular wall motion around the cavity outline. An intervention designed to modify motion in a very localised region and at a specific time in the cardiac cycle has thus been shown to lead to an improvement that was very much greater in extent, both spatially and temporally. If the reverse can occur in disease it suggests that abnormalities of wall movement, particularly those in its timing, may be involved in the genesis of angina in patients with uncomplicated coronary artery disease and that investigation of such abnormalities should be pursued in more detail than before. Indeed, many current approaches to angiographic analysis such as the two-frame or "center line" methods take no account of asynchrony at all. ${ }^{6}$ Detailed analysis of regional wall motion might also be fruitful in other conditions associated with angina and normal coronary arteriograms, such as left ventricular hypertrophy. Regional delay in the onset of inward motion at the start of ejection has already been reported in patients with hypertrophic cardiomyopathy. ${ }^{7}$

Finally, the present case raises the possibility that a direct surgical approach to abnormalities of regional wall motion may be of clinical value in some patients whose coronary artery disease is either too mild or too severe to justify conventional coronary artery surgery. If such direct left ventricular surgery is to develop fruitfully, however, it must be based on a more extensive understanding of the interrelations between regional structure and function than is $\frac{T}{\Phi}$ currently available.

\section{References}

1 Gibson DG, Brown DJ. Abnormal left ventricular wall? movement in patients with chest pain and normalo coronary arteriograms. Relation to inferior $T$ wave $\frac{\bar{\sigma}}{\sigma}$ changes and mitral prolapse. Br Heart J 1979;41:385-® 91.

2 Gibson DG, Prewitt TA, Brown DJ. Analysis of left ${ }^{\hat{\mathcal{C}}}$ ventricular wall movement during isovolumic relaxa- $\vec{O}$ tion and its relation to coronary artery disease. $\mathrm{Br}$ Heart $J$ 1976;38:1010-9.

3 Hammermeister KE, Gibson DG, Hughes D, and Participants in Veterans Administration Cooperative Study on Valvular Heart Disease. Regional variation? in the timing and extent of left ventricular wall motion in normal subjects. Br Heart $J$ 1986;56:226-35.

4 Dawson JR, Gibson DG. Asynchronous left ventricular wall motion during pacing-induced angina $\triangle$ [Abstract]. Circulation 1986;74(suppl 2):360.

5 Marcus ML, Doty DB, Hiratzka LF, Wright CB, Eastham CL. Decreased coronary reserve: a mechanism for angina pectoris in patients with aortic stenosis $\overrightarrow{0}$ and normal coronary arteries. $N$ Engl J Med ${ }_{\infty}^{\infty}$ 1982;307:1362-6.

6 Marier DL, Gibson DG. Limitations of two frameo method for displaying regional left ventricular wall motion in man. Br Heart J 1980;44:555-9.

7 Gibson DG, Sanderson JE, Traill TA, Brown DJ, Goodwin JF. Regional left ventricular wall movement $\mathbb{Q}$ in hypertrophic cardiomyopathy. $\mathrm{Br}$ Heart $J$ 1978;40:1327-33. 